BMJ Paediatrics Open

\section{Cognitive-behavioural therapy combined with music therapy for chronic fatigue following Epstein-Barr virus infection in adolescents: a feasibility study}

To cite: Malik S, Asprusten TT, Pedersen M, et al. Cognitivebehavioural therapy combined with music therapy for chronic fatigue following Epstein-Barr virus infection in adolescents: a feasibility study. BMJ Paediatrics Open 2020;4:e000620. doi:10.1136/ bmjpo-2019-000620

- Additional material is published online only. To view please visit the journal online (http://dx.doi.org/10.1136/ bmjpo-2019-000620).

Received 3 December 2019 Revised 10 March 2020 Accepted 18 March 2020
Check for updates

C Author(s) (or their employer(s)) 2020. Re-use permitted under CC BY-NC. No commercial re-use. See rights and permissions. Published by BMJ.

For numbered affiliations see end of article.

Correspondence to Professor Vegard Bruun Wyller; v.b.b.wyller@medisin.uio.no

\section{ABSTRACT}

Background Cognitive-behavioural therapy (CBT) is effective in chronic fatigue syndrome. However, CBT has not been investigated in postinfectious chronic fatigue (CF), nor is it known whether addition of therapeutic elements from other disciplines might be feasible. We studied the feasibility of a combined CBT and music therapy intervention for CF following Epstein-Barr virus (EBV) infection in adolescents.

Methods Adolescents (12-20 years old) participating in a postinfectious cohort study who developed CF 6 months after an acute EBV infection were eligible for the present feasibility study. A combined CBT and music therapy programme (10 therapy sessions and related homework) was compared with care as usuar in a randomised controlled design. Therapists and participants were blinded to outcome evaluation. Endpoints included physical activity (steps/day), symptom scores, recovery rate and possible harmful effects, but the study was underpowered regarding efficacy. Total follow-up time was 15 months. Results A total of 43 individuals with postinfectious CF were included (21 intervention group, 22 control group). Seven individuals left the study during the first 3 months, leaving 15 in the intervention group and 21 in the control group at 3 months' follow-up. No harmful effects were recorded, and compliance with appointment was high. In intention-to-treat analyses, number of steps/day tended to decrease (difference $=-1158,95 \% \mathrm{Cl}-2642$ to 325 ), whereas postexertional malaise tended to improve (difference $=-0.4,95 \% \mathrm{Cl}-0.9$ to 0.1 ) in the intervention group at 3 months. At 15 months' follow-up, there was a trend towards higher recovery rate in the intervention group (62\% vs $37 \%$ ).

Conclusion An intervention study of combined CBT and music therapy in postinfectious $\mathrm{CF}$ is feasible, and appears acceptable to the participants. The tendencies towards positive effects on patients' symptoms and recovery might justify a full-scale clinical trial.

Trial registration number NCT02499302.

\section{INTRODUCTION}

Chronic fatigue (CF), defined as substantial fatigue lasting for more than 6 months, is a

\section{What is known about the subject?}

Cognitive-behavioural therapy (CBT) is effective and not harmful in chronic fatigue syndrome (CFS).

CBT has not been investigated in postinfectious chronic fatigue (CF).

Combined CBT and music therapy has neither been investigated in CFS nor in postinfectious CF.

\section{What this study adds?}

Combined CBT and music therapy is feasible and acceptable in adolescent postinfectious CF.

- Combined CBT and music therapy may improve recovery and symptoms of postexertional malaise, justifying a full-scale trial.

common complaint after certain infections, such as Epstein-Barr virus (EBV) infection. ${ }^{12}$ If accompanied by other symptoms, such as exertion intolerance, chronic pain and cognitive impairments, the patient might fulfil one of the diagnostic criteria for chronic fatigue syndrome (CFS). CFS is a major cause of disability among adolescents with an estimated prevalence of $0.1 \%-1.0 \% .^{34}$

Evidence suggests a beneficial effect of cognitive-behavioural therapy (CBT) in adults ${ }^{5}$ and adolescents ${ }^{6}$ with CFS, as well as in CF from welldefined causes such as cancer ${ }^{7}$ and diabetes. ${ }^{8}$ Generally, harmful effects of CBT have never been consistently reported; however, effect sizes are usually moderate, and there is a need to establish more effective treatment programmes. Furthermore, it is still unclear to what extent different subgroups respond differently to $\mathrm{CBT}^{9}$; in particular, no study has specifically explored the effect of CBT in postinfectious CF and CFS. 


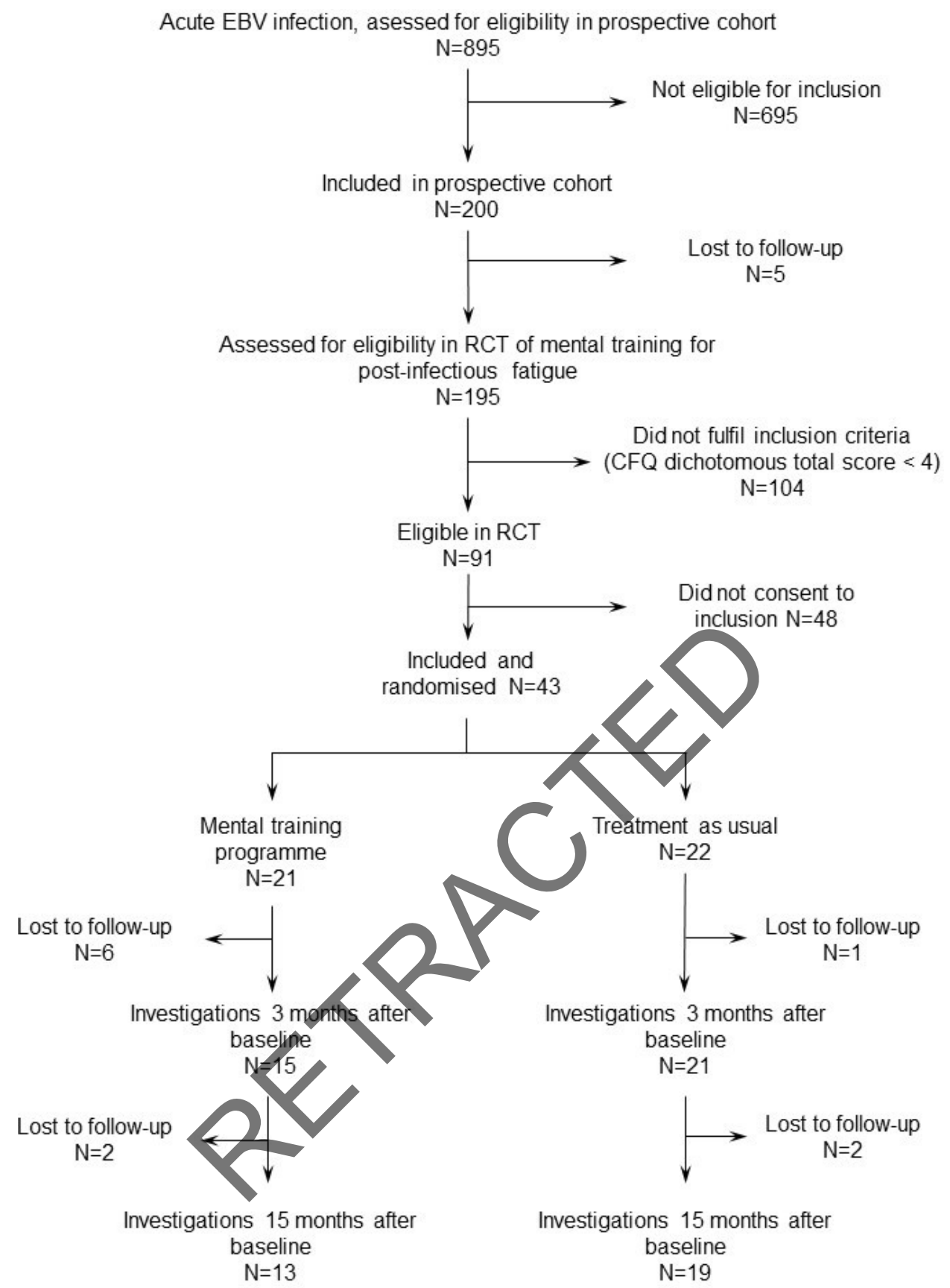

Figure 1 Flow chart of study participants. CFQ, Chalder Fatigue Questionnaire; EBV, Epstein-Barr virus; RCT, randomised controlled trial.

Preliminary evidence suggests that a multidisciplinary approach combining CBT with other elements might improve effectiveness as compared with CBT alone in patients suffering from CFS. ${ }^{10-13}$ Music therapy is an evidence-based complementary therapy form used in many different clinical contexts. ${ }^{14} 15$ Embodiment, emotional and relational experiences in a non-verbal medium, and regulation of arousal are core elements in clinical music therapy. There are no existing studies of music therapy in $\mathrm{CF}$ or CFS, but it has been proven effective in patients with related problems such as fibromyalgia ${ }^{16}$ and long-term sick leave due to stress. ${ }^{17}$
Based on these considerations, we have developed a mental training programme merging elements from music therapy with elements from CBT. The aim of the present study was to explore the feasibility of this mental training programme in adolescents suffering from CF after acute EBV infection, and to provide preliminary estimates of effects as a basis for a full-scale clinical trial in the future. Outcome measures included physical activity, symptoms (such as fatigue, postexertional malaise and pain), recovery from $\mathrm{CF}$ and harmful effects of the training programme. 


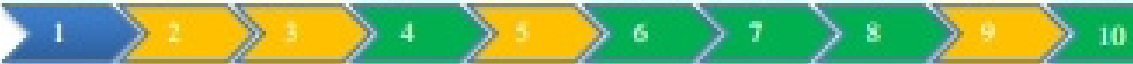

Introductory session

Music Therapy sessions

CBT sessions

Figure 2 Graphical depiction of the 10 therapy sessions in the mental training programme. CBT, cognitive-behavioural therapy.

\begin{tabular}{|c|c|c|c|}
\hline & \multicolumn{2}{|l|}{ Included $(n=43)$} & \multirow[b]{2}{*}{ Eligible, not included $(n=48)$} \\
\hline & Treatment $(n=21)$ & Control $(n=22)$ & \\
\hline \multicolumn{4}{|l|}{ Sex, n (\%) } \\
\hline Male & $4(19)$ & $6(27)$ & $14(29)$ \\
\hline Female & $17(81)$ & $16(73)$ & $34(71)$ \\
\hline Age (years), mean (SD) & $17.7(1.4)$ & $16.9(1.7)$ & $17.4(1.4)$ \\
\hline BMI $\left(\mathrm{kg} / \mathrm{m}^{2}\right)$, mean (SD) & $22.5(3.3)$ & $21.6 k$ & $22.1(2.7)$ \\
\hline \multicolumn{4}{|l|}{ Adheres to Canada 2003 criteria, n (\%) } \\
\hline Yes & $3(14)$ & & $9(19)$ \\
\hline No & $18(86)$ & & $39(81)$ \\
\hline \multicolumn{4}{|l|}{ Adheres to Fukuda criteria, n (\%) } \\
\hline Yes & $5(24)$ & $11(50)$ & $10(21)$ \\
\hline No & $16(76)$ & $11(50)$ & $38(79)$ \\
\hline \multicolumn{4}{|c|}{ Epstein-Barr virus (EBV) load, copies in blood, $n$ (\%) } \\
\hline Negative $(<160)$ & & $13(65)$ & $22(49)$ \\
\hline Low (1600-2000) & & $5(25)$ & $14(31)$ \\
\hline Moderate/high (>2000) & & $2(10)$ & $9(20)$ \\
\hline EBV-VCA-IgG, titre, median (IQR) & 52( & $181(212)$ & $175.5(198)$ \\
\hline $\begin{array}{l}\text { Blood cytotoxic T cell (CD8+) count } \\
\left(10^{9} \text { cells } / L\right) \text {, mean (SD) }\end{array}$ & $0.68(0.25)$ & $0.65(0.23)$ & $0.63(0.25)$ \\
\hline \multicolumn{4}{|l|}{ Efficacy variables } \\
\hline $\begin{array}{l}\text { Chalder Fatigue Questionnaire (CFQ), } \\
\text { total sum score, mean (SD) }\end{array}$ & $20.0(3.3)$ & $19.4(2.9)$ & $19.1(4.8)$ \\
\hline Postexertional malaise, score, mean (SD) & $2.9(1.5)$ & $2.8(1.3)$ & $2.7(1.2)$ \\
\hline $\begin{array}{l}\text { Brief Pain Inventory (BPI), total sum } \\
\text { score, mean (SD) }\end{array}$ & $10.4(5.2)$ & $13.1(4.5)$ & $11.5(5.5)$ \\
\hline $\begin{array}{l}\text { Karolinska Sleep Questionnaire (KSQ), } \\
\text { total sum score, mean (SD) }\end{array}$ & $53.0(14.9)$ & $45.6(10.7)$ & $52.0(13.4)$ \\
\hline $\begin{array}{l}\text { Hospital Anxiety and Depression Scale } \\
\text { (HADS), total sum score, mean (SD) }\end{array}$ & $13.1(5.8)$ & $16.5(6.5)$ & $12.1(6.2)$ \\
\hline $\begin{array}{l}\text { Pediatric Quality of Life Inventory } \\
\text { (PedsQL), total average score, mean (SD) }\end{array}$ & $67(14)$ & $62(15)$ & $70(18)$ \\
\hline $\begin{array}{l}\text { Functional Disability Inventory, total sum } \\
\text { score, mean (SD) }\end{array}$ & $12.2(8.8)$ & $13.7(8.3)$ & $10.1(10.9)$ \\
\hline Steps/day (n), mean (SD) & 7998 (4598) & $8376(3119)$ & 9255 (3795) \\
\hline
\end{tabular}

$\mathrm{P}$ values are based on $\chi^{2}$ test, Fisher's exact test, Student's t-test or Mann-Whitney test, as appropriate.

BMI, body mass index; VCA, viral capsid antigen.

\section{METHODS}

Design overview

The project entitled Chronic Fatigue Following Acute

Epstein-Barr Virus Infection in Adolescents (CEBA) encompasses a prospective postinfectious cohort study followed by a feasibility study of a mental training programme in those participants who developed CF (figure 1). The overall design of CEBA has been 
described elsewhere. ${ }^{18}$ In short, from March 2015 until November 2016, EBV-infected individuals fulfilling the following criteria were assessed for eligibility in the postinfectious cohort study: (A) a serological pattern indicating acute EBV infection; (B) age between 12 and 20 years; and $(\mathrm{C})$ living in one of the Norwegian counties Oslo, Akershus or Buskerud. Exclusion criteria were (A) more than 6 weeks since debut of symptoms suggesting acute EBV infection; (B) any chronic disease that needed regular use of medication; and $(\mathrm{C})$ pregnancy.

A total of 200 adolescents with acute EBV infection were included and followed for 6 months in the postinfectious cohort study. A total of 195 participants attended the 6 months' follow-up visit and were classified as non-CF or CF (dichotomised response $<4$ or $\geq 4$, respectively, on the Chalder Fatigue Questionnaire $(\mathrm{CFQ}))^{19}$

The CF cases $(n=91)$ were assessed for eligibility in the separate feasibility study (ClinicalTrials.gov). This study compared a mental training programme combining music therapy and CBT against care as usual within a randomised controlled design, as described in the present paper. The inclusion period of the feasibility study lasted from September 2015 until May 2017; total follow-up time after randomisation was 15 months.

Participation in the feasibility study was based on informed consent, and thorough information both orally and in writing was provided to the participants and (if younger than 16 years) to their parents guardians.

\section{Investigational programme}

At all encounters in CEBA, participants were subjected to a standardised investigational programme encompassing clinical assessment as well as biomarker sampling. A comprehensive overview is provided elsewhere ${ }^{18}$; here, only investigations relevant for the present feasibility study are detailed.

Physical activity was monitored by the activPAL accelerometer device (PAL Technologies, Glasgow, Scotland) during 7 consecutive days. The accelerometer was attached in the anterior midline on the participants' thigh with custom-made, waterproof adhesive tape. The participants were instructed to wear the device at all times, and only take it off when the recording period was finished. The activPAL provides reliable data on both steps and position $^{20}$; in the present study, the mean number of steps per day was selected as the primary endpoint.

Symptoms were charted by a composite questionnaire encompassing six different instruments; in the entire postinfectious cohort, Cronbach's alpha for these instruments ranged from 0.75 to 0.94 , which was considered acceptable. The $\mathrm{CFQ}^{19}$ charts subjective experience of physical and mental fatigue, has shown acceptable validity among adolescents ${ }^{21}$ and has been translated and validated for a Norwegian population. ${ }^{22}$ It consists of 11 items scored on 4-point Likert scales; in the present study, dichotomous scoring (each item scored 0-0-1-1) was used for definition of fatigue caseness, ${ }^{5}$ whereas ordinal scoring (0-1-2-3) was applied in the analyses of efficacy. The symptom of postexertional malaise, often considered a hallmark of CFS, ${ }^{3}$ was charted with one single item ('How often do you experience more fatigue the day after an exertion?') and scored 1-5 on a Likert scale; higher scores imply more severe symptom burden. The Brief Pain Inventory (BPI) uses four items to assess pain severity ${ }^{23}$; each item is scored on 10-point Likert scales, and the total sum score (range from 0 to 40 ) is reported in the present paper. BPI is validated for adolescents. ${ }^{24}$ The Karolinska Sleep Questionnaire is a validated questionnaire charting insomnia and other sleep disturbances ${ }^{25}$ and has previously been applied in research on adolescent fatigue. ${ }^{26}$ It consists of 14 items scored $1-6$ on Likert scales; the total sum score (range from 14 to 84 ) is reported in the present paper, and lower scores imply more symptoms of sleep problems. The Hospital Anxiety and Depression Scale charts symptoms of depression and anxiety and is validated for adolescents ${ }^{27}$; it consists of 14 items scored $0-3$ on Likert scales, and total sum score (range from 0 to 42 ) is reported in the present paper. The Pediatric Quality of Life Inventory is translated and validated for the Norwegian population. ${ }^{29}$ A total of 23 items are scored on 5-point Likert scales, each point valued $0,25,50,75$ or 100 ; here, the mean score (range $0-100)$ is reported, higher scores indicate better quality of life. Functional Disability Inventory measures disability rełated to everyday activities, and has been thoroughly validated in different adolescent populations. ${ }^{30} 31$ It consists of 15 items scored on 5-point Likert scales; total sum score (range from 0 to 60 ) is applied, where higher scores suggest stronger disability.

Possible side effects related to the mental training programme were charted by a separate set of items using 5-point Likert scales; the answers were dichotomised (present vs not present) prior to analyses. In addition, the questionnaire contained items related to the different diagnostic criteria of CFS as well as simple questions on demographics.

\section{Randomisation and blinding}

Participants were randomised to either mental training or care as usual in a 1:1 probability by a computerbased routine for block randomisation; block size varied randomly between 4 and 6 . It was not possible to blind for treatment. Endpoint evaluation was concealed from patients and therapists, and allocation concealment was ensured using sequentially numbered, opaque, sealed envelopes.

\section{The mental training programme and care as usual}

The intervention consisted of a 10-week mental training programme offered to the relevant individuals as soon as possible after inclusion and randomisation. The programme encompassed one introductory session followed by nine individual therapy sessions (one per 
Table 2 Protocol deviations

\begin{tabular}{|c|c|c|c|c|c|c|c|c|}
\hline \multirow{3}{*}{ Lost to follow-up } & \multicolumn{2}{|c|}{ Treatment } & \multicolumn{2}{|c|}{ Control } & \multicolumn{2}{|c|}{ Treatment } & \multicolumn{2}{|c|}{ Control } \\
\hline & \multicolumn{4}{|c|}{3 months after baseline } & \multicolumn{4}{|c|}{15 months after baseline } \\
\hline & n & $\%$ & $\mathbf{n}$ & $\%$ & $\mathbf{n}$ & $\%$ & $\mathbf{n}$ & $\%$ \\
\hline No & 15 & 71 & 21 & 95 & 13 & 87 & 19 & 90 \\
\hline Yes & 6 & 29 & 1 & 5 & 2 & 13 & 2 & 10 \\
\hline \multicolumn{9}{|c|}{ Interruption of therapy } \\
\hline No & 15 & 71 & n.a & n.a. & n.a. & n.a & n.a. & n.a. \\
\hline Yes* & 6 & 29 & n.a. & n.a. & n.a. & n.a & n.a. & n.a. \\
\hline \multicolumn{9}{|c|}{ Diagnosed with another chronic disorder } \\
\hline No & 15 & 100 & 21 & 100 & 13 & 100 & 19 & 100 \\
\hline Yes & 0 & 0 & 0 & 0 & 0 & 0 & 0 & 0 \\
\hline \multicolumn{9}{|c|}{ Experienced severe illness/trauma } \\
\hline No & 15 & 100 & 21 & 100 & 13 & 100 & 19 & 100 \\
\hline Yes & 0 & 0 & 0 & 0 & 0 & 0 & 0 & 0 \\
\hline \multicolumn{9}{|c|}{ Receiving other therapy for chronic fatigue } \\
\hline No & 15 & 100 & 21 & 100 & 12 & 92 & 19 & 100 \\
\hline Yes & 0 & 0 & 0 & & 1 & 8 & 0 & 0 \\
\hline
\end{tabular}

*The six individuals who interrupted therapy were the same individuals who were lost to follow-up in the intervention group. n.a., not applicable.

week) of 1.5 hours' duration and related homework, combining elements from CBT and music therapy (figure 2). The introductory session was carried out by a researcher, a music therapist and a cognitive therapist, with both the patient and his/her parents/guardians present. In this first session, personal experiences were also shared by a young adult voluntary patient who had himself recovered from CFS. Of the following nine treatment sessions, four were given by a music therapist (sessions 2, 3, 5 and 9) and five were given by a cognitive therapist (sessions 4, 6, 7, 8 and 10. At three of the sessions with cognitive therapists (sessions 4,7 and 10), parents/guardians had the option to participate if the therapist considered it advantageous and the adolescent consented.

Normally, neither general practitioners nor paediatricians in Norway schedule appointment with patients with postinfectious $\mathrm{CF}$ unless they have strongly reduced physical function. Thus, 'care as usual' implies that the relevant individuals would not receive any healthcare for their CF condition in the follow-up period apart from the follow-up visits in the present study.

\section{Therapists, techniques and principles in the mental training programme}

The mental training of all participants allocated to the intervention group was carried out by two therapists only: a child and adolescent psychiatrist holding a specialty in CBT and a music therapist affiliated with the Centre for Research in Music and Health at the Norwegian College of Music. Both of them had previous experiences with adolescents suffering from $\mathrm{CF}$ and other somatic health complaints. They participated in developing the techniques and principles of the present study; no further trainng was provided, but other experienced cognitive therapists and music therapists were available for supervision and discussion throughout the study period.

The first individual sessions in the mental training programme were used to chart activity and sleep patterns, altered functional abilities, withdrawal from social life and changed family dynamics. Thereafter, a variety of therapeutic principles were applied, such as psychoeducation, instructions in techniques for relaxation and visualisation by using music, challenging of automatic thoughts (primarily related to stimulus and outcome expectancies) and behavioural 'experiments' ${ }^{32}$ Together with the patient, the therapist sought to identify negative thoughts and feelings, and to motivate for mental effort/activity to control such negative thoughts and feelings. Cognitive therapy sessions typically blend content and process issues to help make effective changes in attitudes, beliefs and expectations. Collaborative empiricism, guided discovery and the Socratic method provide a comprehensive framework for the processes involved in therapy, while remaining aligned with the core concepts of cognitive therapy. ${ }^{33}$ A central principle throughout the training programme was to achieve therapeutic alliance, which may have a strong impact on treatment effect.

The treatment programme assumes active participation from the patient between the sessions, and the therapists tried to communicate the necessity of individual effort. Parents/guardians are the most essential caregivers for the patient, and in a positive way can motivate the patient and provide guidance through the principles and specific techniques which were introduced in the treatment programme. At the same time, parents/guardians may also strengthen a negative illness behaviour and 
disease attribution, which in turn may have a negative impact on the prognosis. Therefore, it was of great importance to involve the parents/guardians in the treatment programme. Therapist fidelity was ensured by a protocol (cf online supplementary material) detailing the content of every therapy session, ${ }^{32}$ as well as close supervision from senior research collaborators.

As compared with existing CBT protocols for CFS, ${ }^{5} 6$ the present mental training programme deviated mainly on the following points:

- Music therapy was integrated with traditional cognitive techniques.

- Emotions were focused to a greater extent, and specific techniques (such as mindful presence and visualisation) were introduced to increase access to positive feelings.

- Unconscious/automatic experiences were given more attention. Consequently, a fixed plan for graded activity was not an integrated part in the programme, whereas spontaneous experiences not involving conscious planning were encouraged.

- There was an individual adaption regarding specific psychological issues, and parents/guardians were included in some of the treatment sessions.

\section{Effect monitoring}

Assessment of beneficial and possible harmful effects was carried out 3 months after inclusion (ie, immediately after completion of the mental training programme) and 15 months after inclusion. Endpoints included steps/ day count during 7 consecutive days, symptoms (such as fatigue, postexertional malaise, pain) and quality of life/ functional capacity. Recovery was defined as being classified as non-CF (ie, dichotomised response $<4$ on the CFQ).

\section{Patient and public involvement}

Representatives from the patient organisation 'Recovery Norway' as well as representatives from the 'Youth Council' at the Department of Paediatrics and Adolescent Medicine, Akershus University Hospital, Norway, were involved in the design of the present study. Also, we received input from a group of adolescent CFS/myalgic encephalomyelitis sufferers and their next of kin through a pilot test of modified CBT. We carefully assessed the burden of the trial interventions on patients. We intend to disseminate the main results to the trial participants, as well as to relevant patient organisations and other stakeholders.

\section{Power considerations and statistical analyses}

As this was a feasibility study, a formal power calculation was not considered necessary. It should be noted, though, that in a previous research project from our institution, CFS adolescents had a mean (SD) steps/day count of approximately 4500 (2400), and a mean (SD) CFQ total score of $19.1(6.3) .{ }^{34}$ This given, more than
120 participants would be needed in order to detect a moderate effect size (Cohen's $\mathrm{d} \approx 0.5$ ), such as an increase in steps/day of 1200 or a reduction in CFQ total score of 3 $(\alpha=0.05, \beta=0.2)$. In the present study, the total number of eligible individuals was 91 (defined as CF cases 6 months after acute EBV infection), and only 43 consented to inclusion. Hence, the study was strongly underpowered regarding efficacy.

The 'full analysis set' (all randomised participants) was used for intention-to-treat analyses of efficacy. Missing values were imputed using the principle of last observation carried forward. In addition, a non-imputed data set was used for per-protocol analyses. Continuous variables were reported as means or medians, as appropriate. Categorical variables were reported as numbers and percentages. General linear models (analysis of covariance) were used for analyses of treatment effect; the baseline values of each efficacy endpoint were included as covariates. Safety data were summarised descriptively through appropriate data tabulations and descriptive statistics.

\section{RESULTS}

A total of 91 individuals from the postinfectious cohort study were classified as CF cases and thus eligible for inclusion in the present feasibility study (figure 1). More than half of the eligible individuals $(n=48)$ did not consent to participation, mainly because they were concerned over school absence due to therapy sessions, leaving a total of 43 individuals for inclusion and randomisation; 21 were allocated to the intervention (mental training) arm, and 22 to the control (care as usual) arm. Background characteristics and baseline efficacy variables were similar between the included and the non-included groups (table 1).

A total of seven individuals were lost to follow-up during the first 3 months of the study. Six of these individuals were allocated to the intervention group, and they all left the study prior to or immediately after therapy start-up, again reporting concern over school absence as the main reason. Thus, there was a total of 15 participants in the intervention group and 21 in the control group for endpoint evaluation at 3 months' follow-up. No other protocol deviations were registered during the first 3 months of the study (table 2). Of the 15 individuals completing the mental training programme, the number of attended therapy sessions divided by the total number of therapy sessions was 99\% (one music therapy session missing in one individual); thus, compliance with appointments was high among those who stayed in the programme. We do not have data on compliance with recommended homework in-between appointments.

There was a trend towards lower number of steps/day as well as an improvement of postexertional malaise in the intervention group at 3 months (table 3, figure 3). The development of most other symptom scores tended to favour the intervention group; however, effects were small.

An additional four individuals were lost to follow-up during the upcoming year, leaving a total of 13 in the 
Table 3 Outcome of the mental training programme intervention

\begin{tabular}{|c|c|c|c|c|}
\hline \multirow{3}{*}{ CFQ-total sum score } & \multicolumn{2}{|c|}{3 months after baseline } & \multicolumn{2}{|c|}{15 months after baseline } \\
\hline & \multirow[t]{2}{*}{ Intention to treat } & \multirow{2}{*}{ Per protocol } & \multirow[t]{2}{*}{ Intention to treat } & \multirow[t]{2}{*}{ Per protocol } \\
\hline & & & & \\
\hline Treatment group, mean & 19.5 & 19.0 & 16.8 & 14.3 \\
\hline Control group, mean & 20.7 & 20.3 & 18.3 & 18.1 \\
\hline Difference $(95 \% \mathrm{Cl})$ & $-1.1(-4.1$ to 1.9$)$ & $-1.4(-5.3$ to 2.6$)$ & $-1.5(-6.1$ to 3.1$)$ & $-3.8(-9.6$ to 2.1$)$ \\
\hline \multicolumn{5}{|c|}{ Postexertional malaise-score } \\
\hline Treatment group, mean & 2.7 & 2.4 & 2.3 & 1.7 \\
\hline Control group, mean & 3.1 & 2.9 & 2.5 & 2.1 \\
\hline Difference $(95 \% \mathrm{Cl})$ & $-0.4(-0.9$ to 0.1$)$ & $-0.5(-1.1$ to 0.01$)$ & $-0.2(-0.8$ to 0.5$)$ & $-0.4(-1.0$ to 0.3$)$ \\
\hline \multicolumn{5}{|l|}{ BPI-total sum score } \\
\hline Treatment group, mean & 11.3 & 10.7 & 12.5 & 12.9 \\
\hline Control group, mean & 11.7 & 12.4 & 10.6 & 11.0 \\
\hline Difference $(95 \% \mathrm{Cl})$ & $-0.4(-2.9$ to 2.2$)$ & $-1.7(-5.1$ to 1.6$)$ & $1.8(-1.0$ to 4.7$)$ & $1.9(-2.5$ to 6.3$)$ \\
\hline \multicolumn{5}{|l|}{ KSQ-total sum score } \\
\hline Treatment group, mean & 47.0 & 44.2 & 45.3 & 41.1 \\
\hline Control group, mean & 46.2 & 47.9 & & 44.6 \\
\hline Difference $(95 \% \mathrm{Cl})$ & $0.8(-8.1$ to 9.7$)$ & $-3.8(-10.9$ to 3.4$)$ & $2.0(-7.4$ to 11.3$)$ & $-3.5(-14.4$ to 7.4$)$ \\
\hline \multicolumn{5}{|l|}{ HADS - total sum score } \\
\hline Treatment group, mean & 15.1 & 13.9 & 12.8 & 10.0 \\
\hline Control group, mean & 15.1 & 14.2 & 13.5 & 12.4 \\
\hline Difference $(95 \% \mathrm{Cl})$ & $-0.1(-2.7$ to 2.5$)$ & $-0.3(-3$ & $-0.7(-3.8$ to 2.5$)$ & $-2.4(-6.0$ to 1.3$)$ \\
\hline \multicolumn{5}{|c|}{ PedsQL-total average score } \\
\hline Treatment group, mean & 65.9 & & 69.0 & 75.9 \\
\hline Control group, mean & 64.0 & 00. & 71.7 & 74.4 \\
\hline Difference $(95 \% \mathrm{Cl})$ & 1.8( & $3,2(-4.1$ to 10.5$)$ & $-2.7(-11.6$ to 6.2$)$ & $1.4(-9.2$ to 12.0$)$ \\
\hline \multicolumn{5}{|l|}{ FDI-total sum score } \\
\hline Treatment group, mean & 12.4 & 11.0 & 10.6 & 7.4 \\
\hline Control group, mean & & 12.8 & 10.4 & 10.7 \\
\hline Difference $(95 \% \mathrm{Cl})$ & & $-1.8(-5.5$ to 1.9$)$ & $0.2(-4.3$ to 4.6$)$ & $-3.3(-9.0$ to 2.4$)$ \\
\hline \multicolumn{5}{|l|}{ Steps per day, $\mathrm{n}$} \\
\hline Treatment group, mean & & 6198 & 7165 & 7540 \\
\hline Control group, mean & 8296 & 8257 & 8039 & 7782 \\
\hline Difference $(95 \% \mathrm{Cl})$ & $-1158(-2642$ to 325$)$ & $-2059(-3698$ to -421$)$ & $-874(-2800$ to 1052$)$ & -242 (-2944 to 2460$)$ \\
\hline
\end{tabular}

All analyses in the intention to treat columns are based on imputation of missing values based on the 'last observation carried forward' principle. Means and differences 3 and 15 months after baseline are estimated from the parameters of the general linear model. Model diagnostics were performed by visual inspection of residual plots.

BPI, Brief Pain Inventory; CFQ, Chalder Fatigue Questionnaire; FDI, Functional Disability Inventory; HADS, Hospital Anxiety and Depression Scale; KSQ, Karolinska Sleep Questionnaire; PedsQL, Pediatric Quality of Life Inventory.

intervention group and 19 in the control group for assessment 15 months after inclusion. There were no clear differences for any outcome variables (table 3, figure 3). However, there was a trend towards higher recovery rate with a total of $8(62 \%)$ recovered individuals in the treatment group, as compared with a total of $5(39 \%)$ recovered individuals in the control group (table 4).

As for possible harmful effects, there were no important differences between the intervention group and the control group (table 5).

\section{DISCUSSION}

This study shows that a mental training programme combining music therapy and CBT for postinfectious $\mathrm{CF}$ is feasible. Also, the study indicates that the mental training programme may improve symptoms and lead to higher recovery rate over time; however, a full-scale clinical trial with sufficient statistical power would be necessary to demonstrate such an effect.

The acceptance of the intervention among the participants is a main determinant of feasibility. In the present 


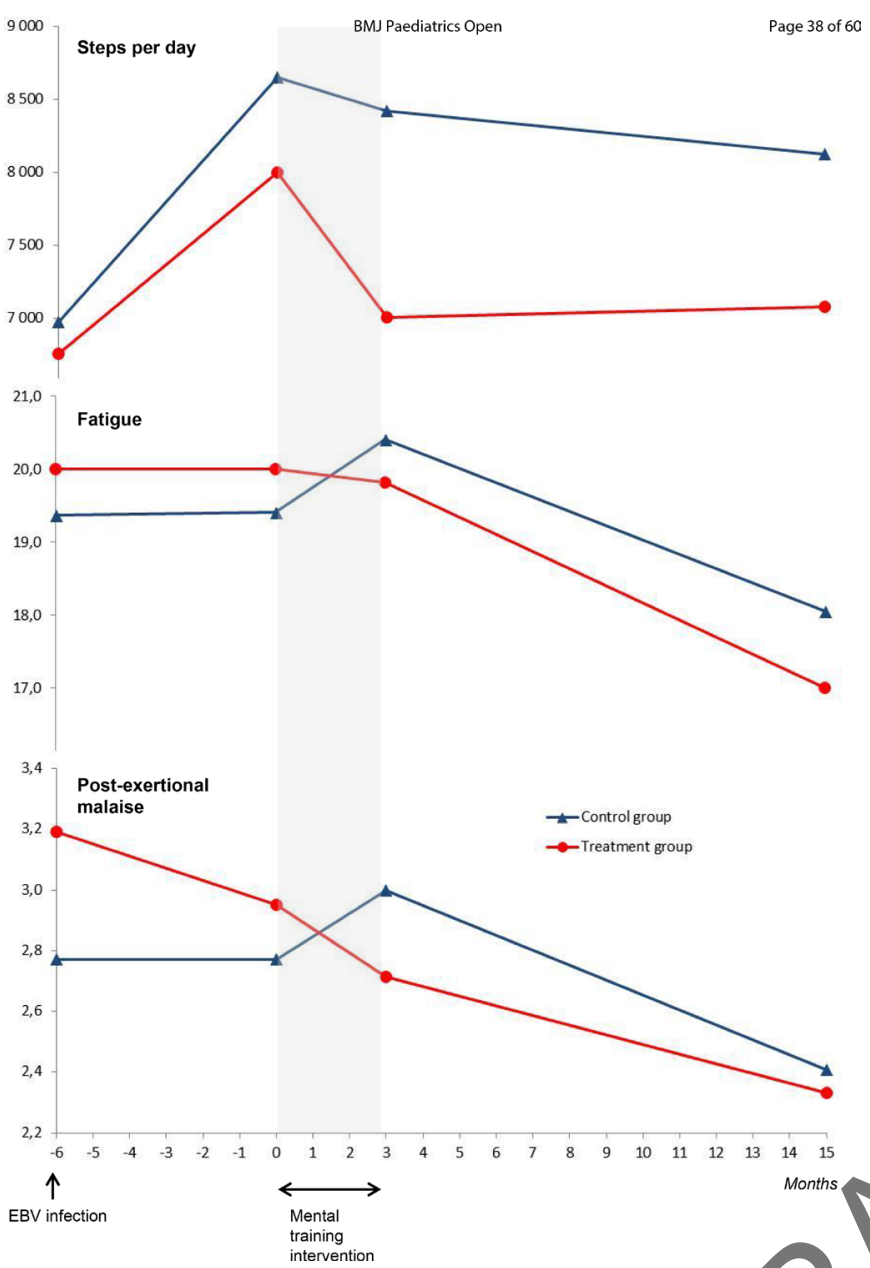

Figure 3 Development of steps/day, fatigue and postexertional malaise over time in the interventiongroup (red) and the control group (blue), based on imputed data sets. The grey area represents the time period in which the mental training programme was provided. EBV, Epstein-Barr virus.

study, the compliance with appointments among those who completed the intervention was high, and the incidence of self-reported adverse events was very low and almost identical among the intervention group and the control group. Taken together, this suggests that the intervention was well tolerated, corroborating results from a recently published qualitative study which reported high levels of satisfaction among those who received the mental training programme. ${ }^{35}$ We cannot rule out that the dropout of six individuals in the intervention group before or immediately after start-up of therapy was due to low tolerability in these participants. However, more probably, this loss to follow-up had the same cause as the poor recruitment of eligible patients into the study. The most likely explanation for these two phenomena is the introduction of a nationwide high school sick leave precept during the study period. This precept initiated public controversies, and-in the context of the present study-caused a lot of concern among the eligible adolescents about participating in a mental training programme that would inevitably result in some school absence. This underlines the importance of considering similar contextual hindrances in the event of a future full-scale clinical trial. Furthermore, given this explanation, we assume the risk of strong selection bias to be rather low, as is supported from the lack of differences between the included and the non-included groups for background and efficacy variables.

Most CBT treatment protocols for CF and CFS encompass an element of increased physical activity over time, ${ }^{56}$ and graded exercise therapy (GET) in itself is shown to have beneficral effects in CFS. ${ }^{36} 37$ Our mental training programme did not contain this element, which may explain why physical activity to our surprise actually tended to decline in the intervention group during the reatment period. Interestingly, we observed a concurrent tendency of improvement of postexertional malaise in the intervention group. This is in line with the own experiences of patients with CFS, often reporting an initial worsening of symptoms during physical exercise which in turn might contribute to the strong controversy over studies that recommend GET in patients with CFS. ${ }^{38} 39$ Thus, the therapeutic approach applied in the present study might be more acceptable from a patient perspective, which further adds to its feasibility.

It has been demonstrated that postinfectious CF tends to recover spontaneously; thus, only a small minority of patients develop CFS over time. Accordingly, in the present study, we observed symptom improvement over time in both the intervention group and the control group. Thus, a modest beneficial effect of the mental training programme might have been somewhat 'diluted' by the natural course of the postinfectious CF

Table 4 Number of recovered patients 3 and 15 months after baseline

\begin{tabular}{|c|c|c|c|c|c|c|c|c|}
\hline & \multicolumn{4}{|c|}{3 months after baseline } & \multicolumn{4}{|c|}{15 months after baseline } \\
\hline & \multicolumn{2}{|c|}{ Recovered } & \multicolumn{2}{|c|}{ Not recovered } & \multicolumn{2}{|c|}{ Recovered } & \multicolumn{2}{|c|}{ Not recovered } \\
\hline & $\mathrm{n}$ & $\%$ & $\mathrm{n}$ & $\%$ & $\mathrm{n}$ & $\%$ & $\mathrm{n}$ & $\%$ \\
\hline Treatment group & 3 & 21 & 11 & 79 & 8 & 62 & 5 & 38 \\
\hline Control group & 4 & 20 & 16 & 80 & 7 & 37 & 12 & 63 \\
\hline Total & 7 & 21 & 27 & 79 & 15 & 47 & 17 & 53 \\
\hline
\end{tabular}

Recovery was defined as a total sum score $\leq 3$ on the Chalder Fatigue Questionnaire (each item scored 0-0-1-1), analogous to the case definition of chronic fatigue used as inclusion criterion in the present study. P values are based on Fisher's exact test or $\chi^{2}$ test, as appropriate. 
Table 5 Adverse effects, self-reported

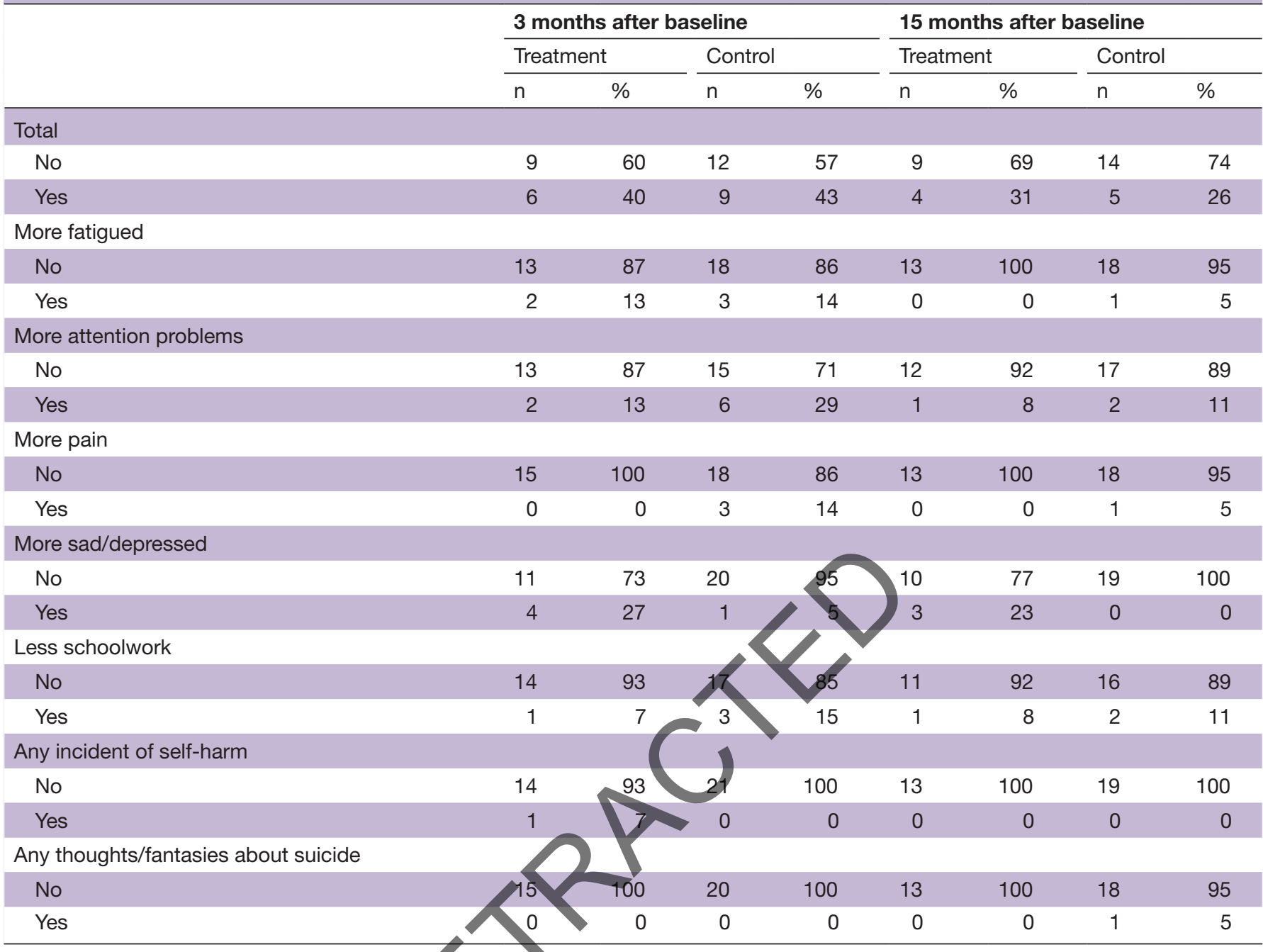

phenomenon. That said, the increased recovery rate after 15 months in the intervention group might indicate that mental training for postinfectious CF is clinically useful. This can only be determined in a full-scale clinical trial. Such a trial should be sufficiently powered to determine the effects in the subgroup of CF individuals who adhere to diagnostic criteria of CFS. The low number of participants in the present study precluded us from performing such subgroup analyses.

\section{Strengths and limitations}

The strengths of this study are the relatively homogeneous group of adolescent participants with the same infectious precipitation of $\mathrm{CF}$ and with no known comorbidities. Weaknesses encompass the lack of formal fidelity checking among the therapists, and the lack of data regarding compliance with recommended homework in-between appointments.

\section{CONCLUSION}

An intervention study of combined CBT and music therapy in postinfectious $\mathrm{CF}$ is feasible, and appears acceptable for the participants. The tendencies towards positive effects on patients' symptoms and recovery might justify a full-scale clinical trial.

Author affiliations

${ }^{1}$ Pediatrics, Akershus University Hospital, Lørenskog, Norway

${ }^{2}$ Clinical Medicine, University of Oslo, Oslo, Norway

${ }^{3}$ Department of Pediatrics and Adolescent Medicine, Drammen Hospital, Drammen, Norway

${ }^{4}$ Center for Music and Health, Norwegian College of Music, Oslo, Norway

${ }^{5}$ Department of Public Health and Nursing, Norwegian University of Science and Technology (NTNU), Trondheim, Norway

Acknowledgements We thank Stine Andersen Ness for invaluable secretary assistance.

Contributors SM, TTA, MP and JM collected clinical data, contributed to study design and participated in data analyses. GT and BvR contributed to study design. ES supervised the statistical analyses. VBW conceived the study, contributed to study design and participated in data analyses. All authors contributed to data interpretation and drafting of the manuscript.

Funding This study was supported by the Health South-East Hospital Trust, Norway.

Competing interests None declared.

Patient consent for publication Not required. 
Ethics approval The study was approved by the Norwegian National Committee for Ethics in Medical Research.

Provenance and peer review Not commissioned; externally peer reviewed.

Data availability statement Data are available upon reasonable request. The data sets used and/or analysed during the current study are available from the corresponding author on reasonable request.

Open access This is an open access article distributed in accordance with the Creative Commons Attribution Non Commercial (CC BY-NC 4.0) license, which permits others to distribute, remix, adapt, build upon this work non-commercially, and license their derivative works on different terms, provided the original work is properly cited, appropriate credit is given, any changes made indicated, and the use is non-commercial. See: http://creativecommons.org/licenses/by-nc/4.0/.

ORCID iD

Vegard Bruun Wyller http://orcid.org/0000-0002-2537-7782

\section{REFERENCES}

1 Hickie I, Davenport T, Wakefield D, et al. Post-infective and chronic fatigue syndromes precipitated by viral and non-viral pathogens: prospective cohort study. BMJ 2006;333:575

2 Katz BZ, Shiraishi Y, Mears CJ, et al. Chronic fatigue syndrome after infectious mononucleosis in adolescents. Pediatrics 2009;124:189-93.

3 IOM Institute of medicine. Beyond myalgic Encephalomyelitis/ Chronic fatigue syndrome: redefining an illness. Washington, DC: The National Academies Press, 2015. http://www.iom.edu/mecfs

4 Royal College of Paediatrics and Child Health. Evidence based guidelines for the management of CFS/ME (chronic fatigue syndrome/myalgic encephalopathy) in children and young adults. London: Royal College of Paediatrics and Child Health, 2004.

5 White PD, Goldsmith KA, Johnson AL, et al. Comparison of adaptive pacing therapy, cognitive behaviour therapy, graded exercise therapy, and specialist medical care for chronic fatigue syndrome (PACE): a randomised trial. Lancet 2011;377:823-36.

6 Nijhof SL, Bleijenberg G, Uiterwaal CSPM, et al. Effectiveness of Internet-based cognitive behavioural treatment for adolescents with chronic fatigue syndrome (FITNET): a randomised controlled trial. Lancet 2012;379:1412-8.

7 Abrahams HJG, Gielissen MFM, Donders RRT, et al. The efficacy of Internet-based cognitive behavioral therapy for severely fatigued survivors of breast cancer compared with care as usual: a randomized controlled trial. Cancer 2017;123:3825-

8 Menting J, Tack CJ, van Bon AC, et al. Web-Based cognitive behavioural therapy blended with face-to-face sessions for chronic fatigue in type 1 diabetes: a multicentre randomised controlled trial. Lancet Diabetes Endocrinol 2017;5:448-5

9 Cella M, Chalder T, White PD. Does the heterogeneity of chronic fatigue syndrome moderate the response to cognitive behaviour therapy? an exploratory study. Psychother Psychosom 2011;80:353-8.

10 Rimes KA, Wingrove J. Mindfulness-based cognitive therapy for people with chronic fatigue syndrome still experiencing excessive fatigue after cognitive behaviour therapy: a pilot randomized study. Clin Psychol Psychother 2013;20:107-17.

11 Viner R, Gregorowski A, Wine C, et al. Outpatient rehabilitative treatment of chronic fatigue syndrome (CFS/ME). Arch Dis Child 2004;89:615-9.

12 Crawley EM, Gaunt DM, Garfield K, et al. Clinical and costeffectiveness of the lightning process in addition to specialist medical care for paediatric chronic fatigue syndrome: randomised controlled trial. Arch Dis Child 2018;103:155-64.

13 Stubhaug B, Lier HO, Aßmus J, et al. A 4-day Mindfulness-Based cognitive behavioral intervention program for CFS/ME. an open study, with 1-year follow-up. Front Psychiatry 2018;9:720.

14 Bradt J, Dileo C, Potvin N. Music for stress and anxiety reduction in coronary heart disease patients. Cochrane Database Syst Rev 2013;12:CD006577.

15 Bradt J, Goodill SW, Dileo C. Dance/movement therapy for improving psychogical and physical outcomes in cancer patients. Cochrane Database Syst Rev 2011;10:CD007103.
16 Torres E, Moe T. Group Music and Imagery (GMI) for Treating Fibromyalgia. In: Grocke D, Moe T, eds. Guided Imagery \& Music (GIM) and Music Imagery Methods for Individual and Group Therapy. London: Jessica Kingsley, 2015: 267-76.

17 Beck BD. Guided imagery and music (Gim) with adults on sick leave suffering from work-related stress. Aalborg university, 2012.

18 Pedersen M, Asprusten TT, Godang K, et al. Predictors of chronic fatigue in adolescents six months after acute Epstein-Barr virus infection: a prospective cohort study. Brain Behav Immun 2019;75:94-100.

19 Chalder T, Berelowitz G, Pawlikowska T, et al. Development of a fatigue scale. J Psychosom Res 1993;37:147-53.

20 Grant PM, Ryan CG, Tigbe WW, et al. The validation of a novel activity monitor in the measurement of posture and motion during everyday activities. Br J Sports Med 2006;40:992-7.

21 Tanaka M, Fukuda S, Mizuno K, et al. Reliability and validity of the Japanese version of the Chalder fatigue scale among youth in Japan. Psychol Rep 2008;103:682-90.

22 Loge JH, Ekeberg O, Kaasa S. Fatigue in the general Norwegian population: normative data and associations. J Psychosom Res 1998;45:53-65

23 Klepstad P, Loge JH, Borchgrevink PC, et al. The Norwegian brief pain inventory questionnaire: translation and validation in cancer pain patients. J Pain Symptom Manage 2002;24:517-25.

24 Engel JM, Kartin D, Carter GT, et al. Pain in youths with neuromuscular disease. Am J Hosp Palliat Care 2009;26:405-12.

25 Akerstedt T, Ingre M, Broman J-E, et al. Disturbed sleep in shift workers, day workers, and insomniacs. Chronobiol Int 2008;25:333-48

26 Pedersen M, Ekstedt M, Småstuen MC, et al. Sleep-Wake rhythm disturbances and perceived sleep in adolescent chronic fatigue syndrome. J Sleep Res 2017;26:595-601.

27 Zigmond AS, Snaith RP. The hospital anxiety and depression scale. Acta Psychiatr Scand 1983;67:361-70.

28 Minalca AM, Pilecka W. The factorial structure and validity of the hospital anxiety and depression scale (HADS) in Polish adolescents. Psychiatr Pol 2015;49:1071-88.

29 Reinfjell T, Diseth TH, Veenstra M, et al. Measuring health-related quality of life in young adolescents: reliability and validity in the Norwegian version of the pediatric quality of life inventory 4.0 (PedsQL) generic core scales. Health Qual Life Outcomes 2006;4:61.

30 Walker LS, Greene JW. The functional disability inventory: measuring a neglected dimension of child health status. J Pediatr Psychol 1991;16:39-58

31 Claar RL, Walker LS. Functional assessment of pediatric pain patients: psychometric properties of the functional disability inventory. Pain 2006;121:77-84.

32 Dept. of Pediatrics. Mental intervention for chronic fatigue syndrome (CFS/ME) following acute Epstein-Barr virus infection. treatment manual for the intervention part of the CEBA-project. Akershus University Hospital, 2015.

33 Tee J, Kazantzis N. Collaborative empiricism in cognitive therapy: a definition and theory for the relationship construct. Clin Psychol Sci Prac 2011;18:47-61.

34 Sulheim D, Fagermoen E, Winger A, et al. Disease mechanisms and clonidine treatment in adolescent chronic fatigue syndrome: a combined cross-sectional and randomized clinical trial. JAMA Pediatr 2014;168:351-60.

35 Trondalen G, Mangersnes J, Bonde LO, et al. Music therapy for chronic fatigue following Epstein-Barr virus infection in adolescents. Music Med 2020

36 Larun L, Brurberg KG, Odgaard-Jensen J, et al. Exercise therapy for chronic fatigue syndrome. Cochrane Database Syst Rev 2016;2:CD003200.

37 Clark LV, Pesola F, Thomas JM, et al. Guided graded exercise self-help plus specialist medical care versus specialist medical care alone for chronic fatigue syndrome (GETSET): a pragmatic randomised controlled trial. Lancet 2017;390:363-73.

38 Kindlon T. Do graded activity therapies cause harm in chronic fatigue syndrome? J Health Psychol 2017;22:1146-54.

39 Vink M, Vink-Niese A. Graded exercise therapy for myalgic Encephalomyelitis/Chronic fatigue syndrome is not effective and unsafe. Re-analysis of a cochrane review. Health Psychol Open 20 18;5:2055102918805187:205510291880518. 